\title{
Droplet Collision Simulation by a Multi-Speed Lattice Boltzmann Method
}

\section{Conference Paper}

\section{Author(s):}

Lycett-Brown, Daniel; Karlin, llya; Luo, Kai H.

Publication date:

2011

Permanent link:

https://doi.org/10.3929/ethz-b-000037906

Rights / license:

In Copyright - Non-Commercial Use Permitted

Originally published in:

Communications in Computational Physics 9(5), https://doi.org/10.4208/cicp.311009.091110s 


\title{
Droplet Collision Simulation by a Multi-Speed Lattice Boltzmann Method
}

\author{
Daniel Lycett-Brown ${ }^{1}$, Ilya Karlin ${ }^{1,2}$ and Kai H. Luo ${ }^{1, *}$ \\ ${ }^{1}$ Energy Technology Group, School of Engineering Sciences, University of \\ Southampton, SO17 1BJ, UK. \\ ${ }^{2}$ Aerothermochemistry and Combustion Systems Lab, ETH Zurich, \\ 8092 Zurich, Switzerland.
}

Received 31 October 2009; Accepted (in revised version) 9 November 2010

Available online 28 January 2011

\begin{abstract}
Realization of the Shan-Chen multiphase flow lattice Boltzmann model is considered in the framework of the higher-order Galilean invariant lattices. The present multiphase lattice Boltzmann model is used in two-dimensional simulation of droplet collisions at high Weber numbers. Results are found to be in a good agreement with experimental findings.
\end{abstract}

AMS subject classifications: 76A02, 76M25, 76T10, 35Q20

Key words: Multiphase flow, lattice Boltzmann method, droplets.

\section{Introduction}

\subsection{The lattice Boltzmann method}

The Lattice Boltzmann method (LBM) is a rapidly developing approach to computational fluid dynamics (CFD). One of its major advantages over traditional CFD is in the modelling of multiphase flows. In this paper the Shan-Chen multiphase method [1] is used to study binary droplet collisions. Numerous improvements to this model have been suggested in the literature, including the use of a different equation of state (EoS) [2], and the increase in the order of isotropy of the force term [3]. The possibilities of combining different methods will be addressed in this paper to improve stability in the droplet collision simulation. These methods are initially combined with the standard LBM. Higher order lattices, derived from an entropic viewpoint $[4,5]$, are then considered.

The LBM originally evolved from Lattice Gas Cellular Automaton (LGCA) methods, which streamed individual particles along lattice velocities. Another starting point for

*Corresponding author. Email addresses: djlb1e08@soton.ac.uk (D. Lycett-Brown), karlin@lav.mavt. ethz.ch (I. Karlin), k.h.luo@soton.ac.uk (K. H. Luo) 
the LBM, used here, is the Boltzmann transport equation, which describes the evolution of the density function for a gas of point like particles

$$
\frac{\partial f}{\partial t}+\boldsymbol{v} \cdot \nabla_{x} f+\boldsymbol{F} \cdot \nabla_{p} f=\left.\frac{\partial f}{\partial t}\right|_{\text {coll }}
$$

where $\boldsymbol{F}$ is an external force. In this work the Bhatnagar-Gross-Krook (BGK) collision term is used on the right hand side of this equation. Discretisation of this equation, details of which can be found in many papers, including [6], leads to the LBM. The method involves the streaming of distribution functions between fixed nodes along lattice velocities $\boldsymbol{v}_{i}$, and then relaxing these distributions to their local equilibrium, $f_{i}^{\mathrm{eq}}(\boldsymbol{x}, t)$, at each lattice node (the forcing term is dropped for the time being),

$$
f_{i}\left(x+v_{i}, t+1\right)-f_{i}(x, t)=-\frac{1}{\tau}\left[f_{i}(x, t)-f_{i}^{\mathrm{eq}}(x, t)\right],
$$

where $\tau$ is the relaxation time. Macroscopic quantities are recovered from moments of the distribution function

$$
\begin{aligned}
& \rho=\sum_{i} f_{i}, \\
& \rho \boldsymbol{u}=\sum_{i} \boldsymbol{v}_{i} f_{i} .
\end{aligned}
$$

These are used to calculate the equilibrium distribution functions at each node. In two spatial dimensions, one commonly used lattice has nine velocities, $(0,0),(0, \pm 1),( \pm 1,0)$ and $( \pm 1, \pm 1)$, and is labeled the D2Q9 lattice. Its equilibrium distribution functions, to second order in velocity, are given by

$$
f_{i}^{\mathrm{eq}}=w_{i} \rho\left(1+\frac{\boldsymbol{v}_{i} \cdot \boldsymbol{u}}{c_{\mathrm{s}}^{2}}+\frac{\left(\boldsymbol{v}_{i} \cdot \boldsymbol{u}\right)^{2}}{2 c_{\mathrm{s}}^{4}}-\frac{u^{2}}{2 c_{\mathrm{s}}^{2}}\right)
$$

where $w_{i}$ is a weighting function, and $c_{\mathrm{S}}$ is the speed of sound related to the reference temperature $T_{0}$ as $c_{\mathrm{s}}^{2}=T_{0}$. This equilibrium is designed in such a way as to reproduce the isothermal Navier-Stokes equations in the macroscopic limit, at the reference temperature $T_{0}=1 / 3$.

An alternative derivation of the LBM comes from considering entropy. Equilibrium distribution functions are derived to minimize a specified entropy function. From this entropic LBM (ELBM) a systematic method has been developed for producing stable, higher order lattices [4]. This method is outlined in Section 3.

\subsection{Droplet collisions}

The study of binary droplet collisions has many important applications across different scientific areas, from understanding cloud formation in climate theory, to engineering applications, such as turbine blade cooling, spray coatings and spray combustion in diesel 


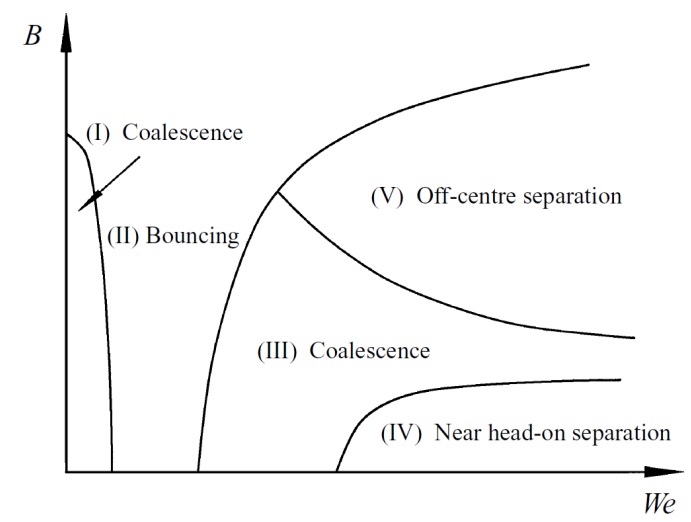

Figure 1: Regimes of binary droplet collision outcomes (from [9]).

internal combustion engines. Modeling of multiphase flows with traditional CFD is challenging as it requires the tracking of the interface between the different phases. This becomes increasingly difficult in regimes where two interfaces split or meet and merge together, as is the case with droplet collisions. The LBM however, does not require the explicit tracking of interfaces, and therefore has no additional difficulty associated with modeling droplet collisions.

Many comprehensive experimental studies of binary droplet collisions are available [7, 8], including one by Qian and Law [9]. Various regimes of droplet collisions are identified by two dimensionless parameters, the Weber number We,

$$
\mathrm{We}=\frac{2 R \rho U^{2}}{\sigma}
$$

where $R$ is the droplet radius (identical for both droplets in this equation), $U$ is their relative velocity and $\sigma$ is the surface tension of the liquid, and the impact parameter $B$,

$$
B=\frac{\chi}{2 R}
$$

where $\chi$ is the separation between the centres of the droplets, perpendicular to their direction of motion. Different regimes for the droplet collisions are found, and a general representation of these regions is given in Fig. 1.

The difficulty in reproducing the results of Fig. 1 with the LBM is associated with reaching the relevant values for the Reynolds number, given by

$$
\operatorname{Re}=\frac{2 R U}{v_{1}}
$$

where $v_{1}$ is the kinematic viscosity of the liquid, and for the density ratio and Weber number. In experiments, these values are typically of the order of a thousand for Reynolds number and density ratio between the phases, and of the order of a hundred for Weber number. 
A number of studies have been done on droplet collisions using the LBM. Inamuro et al. [10] used the projection method [11] applied to the free energy model. They produced results of droplet collision at a Reynolds number of the order 2000 and at Weber numbers up to 100, observing regimes (III), (IV) and (V) with good agreement to theory [7]. However regime (II) is not observed and while it is stated that their method can reach density ratios up to 1000:1, the droplet collision results are only given at 50:1. Luo et al. [12] used the Shan-Chen single component multiphase (SCMP) method with a multiple-relaxationtime scheme. They achieved Reynolds numbers up to a few hundred, and Weber number up to 100 , but also only did so at a density ratio of around 50:1. Regimes (I), (III), (IV) and $(\mathrm{V})$ were observed but regime (II) was not.

To achieve the experimental values of density ratio, increased stability is required. Section 2 outlines the Shan-Chen model, and existing methods of its improvement. Details of the ELBM are given in Section 3, and its application to the Shan-Chen model described in Section 4. How to combine these different methods is discussed, and the results of these combinations are given in Section 5. Finally, conclusions are drawn in Section 6.

\section{Multiphase LBM}

\subsection{The Shan-Chen model}

The original idea for multiphase flow in LBM was based on a two component LGCA model $[13,14]$, and involved two particle distribution functions, each having its own LBGK equation, with an additional term, $S_{i}$, introduced to represent the interaction between the two components

$$
f_{i}\left(x+\boldsymbol{v}_{i}, t+1\right)-f_{i}(x, t)=-\frac{1}{\tau}\left[f_{i}(x, t)-f_{i}^{\mathrm{eq}}(\boldsymbol{x}, t)\right]+S_{i}
$$

Shan and Chen modelled this perturbation term by a force $\boldsymbol{F}_{i}$ (see [1])

$$
S_{i}=\boldsymbol{F}_{i} \cdot \boldsymbol{v}_{i}
$$

derived from an interaction potential

$$
V\left(x, x^{\prime}\right)=G_{\sigma \bar{\sigma}}\left(x, x^{\prime}\right) \psi_{\sigma}(x) \psi_{\bar{\sigma}}\left(x^{\prime}\right),
$$

where $G_{\sigma \bar{\sigma}}$ is a Green's function, which controls the strength of the interaction, and whether it is attractive or repulsive, and $\psi$ is an effective number density. The different fluid components are represented by $\sigma$ and $\bar{\sigma}$. In a SCMP system, this is simplified to

$$
G_{\sigma \bar{\sigma}}\left(x, x^{\prime}\right)=G\left(x, x^{\prime}\right)
$$


This potential produces a change of momentum at each lattice site

$$
\frac{d P}{d t}(\boldsymbol{x})=-\psi(\boldsymbol{x}) \sum_{i} G\left(\boldsymbol{x}, \boldsymbol{x}+\boldsymbol{v}_{i}\right) \psi\left(\boldsymbol{x}+\boldsymbol{v}_{i}\right) \boldsymbol{v}_{i}
$$

resulting in

$$
\rho \boldsymbol{u}^{\prime}=\rho \boldsymbol{u}+\tau \frac{d P}{d t} .
$$

The equilibrium distribution functions are then calculated from Eq. (1.4), using the new velocity $\boldsymbol{u}^{\prime}$. If the nearest neighbour lattice sites only are used in the force calculation, then

$$
G\left(x, x^{\prime}\right)= \begin{cases}G, & \left|x-x^{\prime}\right| \leq\left|v_{i}\right| \\ 0, & \left|x-x^{\prime}\right|>\left|v_{i}\right|\end{cases}
$$

and Eq. (2.5) becomes

$$
\boldsymbol{F}(\boldsymbol{x})=-G \psi(\boldsymbol{x}) \sum_{i} \psi\left(\boldsymbol{x}+\boldsymbol{v}_{i}\right) \boldsymbol{v}_{i}
$$

and can now be approximated by

$$
\boldsymbol{F}(\boldsymbol{x}) \approx-c_{\mathrm{s}}^{2} G \psi(\boldsymbol{x}) \nabla \psi(\boldsymbol{x}) .
$$

Using this form of the interaction force, the resulting EoS of the system becomes

$$
P=\rho c_{\mathrm{s}}^{2}+\frac{G c_{\mathrm{s}}^{2}}{2} \psi(x)^{2},
$$

where the second term on the right hand side alters the ideal gas EoS as a result of the interaction force. With the correct choice of $G$ this term can lead to phase separation. For this phase separation to occur a suitable $\psi(\rho)$ must be chosen, and $G$ must be negative and below a certain critical value. The original choice was [1]

$$
\psi(\rho)=\rho_{0}\left(1-e^{-\frac{\rho}{\rho_{0}}}\right),
$$

where $\rho_{0}$ is a reference density. This is a monotonically increasing function of density and therefore provides the correct form for the pressure to induce phase separation. It also has the advantage that it becomes constant at high density and therefore prevents high density areas collapsing. Improvements to this basic model are discussed in the following section.

\subsection{Grid refinement and isotropy}

One major problem affecting the above multiphase model, and others, is the formation of spurious velocity currents at curved interfaces. These velocities increase with the density ratio, with the resulting instabilities severely limiting the density ratios that can be 
achieved. One current explanation for these velocities lies in the discretisation of the calculation of the force term which lacks sufficient isotropy [15]. The order of isotropy can be increased by relaxing the nearest neighbour constraint in the force term

$$
\boldsymbol{F}=-G \psi(\boldsymbol{x}) \sum_{i} w\left(\left|\boldsymbol{v}_{i}\right|^{2}\right) \psi\left(\boldsymbol{x}+\boldsymbol{v}_{i}\right) \boldsymbol{v}_{i}
$$

where $w\left(\left|\boldsymbol{v}_{i}\right|^{2}\right)$ is a weighting function, different from that used in the calculation of the equilibrium distribution functions, and is introduced here to be equivalent to the function $G\left(x, x^{\prime}\right)$. This can be approximated by

$$
F_{\alpha}=-\frac{G c_{\mathrm{s}}^{2}}{2} \psi \partial_{\alpha} \psi-\frac{G c_{\mathrm{s}}^{4}}{4} \psi \partial_{\alpha} \Delta \psi+\mathcal{O}\left(\partial^{5}\right)
$$

From the definition

$$
\partial_{\beta} P_{\alpha \beta}=-F_{\alpha}+\partial_{\alpha}\left(c_{\mathrm{s}}^{2} \rho\right),
$$

the pressure tensor is now given by

$$
P_{\alpha \beta}=\left(T_{0} \rho+\frac{G T_{0}}{2} \psi^{2}+\frac{G T_{0}^{2}}{4}|\nabla \psi|^{2}+\frac{G T_{0}^{2}}{2} \psi \Delta \psi\right) \delta_{\alpha \beta}-\frac{G T_{0}^{2}}{2} \partial_{\alpha} \psi \partial_{\beta} \psi+\mathcal{O}\left(\partial^{4}\right) .
$$

The velocities $v_{i}$ in Eq. (2.12) can be extended to give any required order of isotropy, with increasing orders decreasing the spurious velocities, and increasing stability $[3,15]$. This allows higher density ratios to be reached, although it does incur some computational overhead.

Another method involves refining the grid, while keeping the surface tension and the density ratio in the system constant. This cannot be done with the original force equation as density and surface tension are not independently tunable. Introducing a second term into the force equation gives [3]

$$
\boldsymbol{F}=-\psi(\boldsymbol{x}) \sum_{i} w\left(\left|\boldsymbol{v}_{i}\right|^{2}\right)\left[G_{1} \psi\left(\boldsymbol{x}+\boldsymbol{v}_{i}\right)+G_{2} \psi\left(\boldsymbol{x}+2 \boldsymbol{v}_{i}\right)\right] \boldsymbol{v}_{i} .
$$

There are now two tunable parameters in the system, $G_{1}$ and $G_{2}$, allowing the EoS, and therefore the density ratio and the surface tension, to be controlled separately. Surface tension is related to the width of the interface and this method allows the interface to be spread over a larger number of interface points, while keeping its width proportional to the size of the system. In this way stability is increased by spreading the density drop over a larger number of grid points. Having introduced this more general equation for the force, the pressure tensor is now given by

$$
P_{\alpha \beta}=\left(T_{0} \rho+\frac{A_{1} T_{0}}{2} \psi^{2}+\frac{A_{2} T_{0}^{2}}{4}|\nabla \psi|^{2}+\frac{A_{2} T_{0}^{2}}{2} \psi \Delta \psi\right) \delta_{\alpha \beta}-\frac{A_{2} T_{0}^{2}}{2} \partial_{\alpha} \psi \partial_{\beta} \psi+\mathcal{O}\left(\partial^{4}\right),
$$

resulting in the surface tension

$$
\sigma=-\frac{A_{2} T_{0}^{2}}{2} \int_{-\infty}^{\infty}\left|\partial_{y} \psi\right|^{2} d y
$$


where

$$
A_{1}=G_{1}+2 G_{2}, \quad A_{2}=G_{1}+8 G_{2}
$$

It can be seen that it is now possible to vary the surface tension and therefore the interface width, while keeping the density ratio constant, by changing $G_{1}$ and $G_{2}$ in such a way as to change $A_{2}$ keeping $A_{1}$ constant.

This idea can be extended so that the interface resolution can be increased, increasing stability, while keeping density ratio and surface tension constant. This is done by slightly modifying Eq. (2.11) to give

$$
\psi(\rho)=\sqrt{\rho_{0}}\left(1-e^{-\frac{\rho}{\rho_{0}}}\right),
$$

and rescaling co-ordinates by

$$
y^{\prime}=\rho_{0} y
$$

It can then be shown [3] that the interface width can be changed by varying $\rho_{0}$, keeping density ratio and surface tension constant, as long as $A_{2}$ is varied according to

$$
A_{2}=\frac{A_{2}^{\prime}}{\rho_{0}^{2}}
$$

The results presented in [3] give a near ten times reduction in velocities for a doubling of the grid size, although each increase in grid size incurs additional computational cost.

Finally, another improvement to the Shan-Chen model was suggested by Yuan and Schaefer [2]. This involved changing the functional form of $\psi$ to achieve a more realistic equation of state. Rearranging Eq. (2.10) gives

$$
\psi(\rho)=\sqrt{\frac{2\left(P-\rho T_{0}\right)}{G T_{0}}} .
$$

In this equation the pressure can be substituted with any form of the EoS, for example the Carnahan-Starling EoS [17]

$$
P=\rho R T \frac{1+\frac{b \rho}{4}+\left(\frac{b \rho}{4}\right)^{2}-\left(\frac{b \rho}{4}\right)^{3}}{\left(1-\frac{b \rho}{4}\right)^{3}}-a \rho^{2} .
$$

Note that with Eq. (2.23) in the force calculation, Eq. (2.12), the parameter $G$ now cancels out, and the phase change is controlled by the temperature in the EoS. The advantage of using the CS EoS is a large increase in the density ratio achievable, with almost no increase in computational time. The liquid to gas density ratio achieved while using this equation is over 1000:1, approaching the density ratio observed in realistic systems. 


\section{Multi-speed lattices from the ELBM}

One limiting factor on both the Weber and Reynolds numbers attainable in a lattice Boltzmann simulation is imposed by the magnitude of the flow velocity achievable. Reynolds number depends linearly on velocity, but Weber number has quadratic dependence, and therefore increases significantly with increases in relative droplet velocity. However, the use of the standard LBM is limited in that respect because of the incomplete Galilean invariance manifested in cubic deviations in higher-order equilibrium moments. This restricts the velocities in simulations to $u<0.1$, which, in particular, causes a severe limitation on the Weber number in the droplet impact simulation. It is therefore useful to investigate LBMs that can support higher values of the flow velocity.

Recently $[4,5]$ a systematic derivation of higher-order lattices suitable for hosting lattice Boltzmann models has been established. In particular, higher-order complete Galilean invariant LB models (free of the cubic error of the standard LB) have been suggested in any spatial dimension. In this paper, we use the D2Q25 lattice in two dimensions, established as a tensor product of two copies of one-dimensional velocity sets $\{0, \pm 1, \pm 3\}$. It was shown in $[4,5]$ that the D2Q25 lattice admits two non-equivalent LB models corresponding to the reference temperatures

$$
T_{0}=1 \mp \sqrt{\frac{2}{5}}
$$

The weights of the velocities in one dimension, corresponding to the above two values of the reference temperature are

$$
W_{0}=\frac{4}{45}(4 \pm \sqrt{10}), \quad W_{1}=\frac{3}{80}(8 \mp 10), \quad W_{3}=\frac{1}{720}(16 \mp 5 \sqrt{10}) .
$$

To extend this lattice to two dimensions velocities are obtained from the tensor product of the one dimensional lattice with itself. Weights are similarly obtained from products of the one dimensional weights, and in this case are given by algebraic products of corresponding one-dimensional weights

$$
\begin{aligned}
& W_{(0,0)}=W_{0} W_{0}, \\
& W_{(0, \pm 1)}=W_{( \pm 1,0)}=W_{0} W_{1}, \\
& W_{(0, \pm 3)}=W_{( \pm 3,0)}=W_{0} W_{3}, \\
& W_{( \pm 1, \pm 1)}=W_{1} W_{1}, \\
& W_{( \pm 1, \pm 3)}=W_{( \pm 3, \pm 1)}=W_{1} W_{3}, \\
& W_{( \pm 3, \pm 3)}=W_{3} W_{3} .
\end{aligned}
$$

In the sequel, we use the higher reference temperature which corresponds to the larger value of the speed of sound. This choice is motivated by the fact that we are interested in 
simulating droplet collisions at larger Weber numbers. Finally, the equilibrium populations are used in the form

$$
f_{i}^{\mathrm{eq}}=W_{i}\left[1+\frac{v_{i \alpha} u_{\alpha}}{T_{0}}+\frac{u_{\alpha} u_{\beta}}{2 T_{0}^{2}}\left(v_{i \alpha} v_{i \beta}-T_{0} \delta_{\alpha \beta}\right)+\frac{u_{\alpha} u_{\beta} u_{\gamma}}{6 T_{0}^{3}} v_{i \gamma}\left(v_{i \alpha} v_{i \beta}-3 T_{0} \delta_{\alpha \beta}\right)\right] .
$$

The D2Q25 LB model just described is fully Galilean invariant (unlike the D2Q9 model), allowing for larger values of the velocity to be used in a simulation, and admits the entropy function which is a prerequisite for stability.

\section{Applying multi-speed lattices to the Shan-Chen model}

Having established a stable, higher order LBM, it is interesting to investigate its effect on the stability of the Shan-Chen model. In the D2Q9 model, the nine velocities of the lattice were also used in the finite difference scheme for evaluating the force at each lattice point. It was shown in Section 2.2 that the order of isotropy of this calculation could be increased by considering a larger set of velocities. However, for the D2Q25 lattice, only the lattice velocities are used in the force calculation.

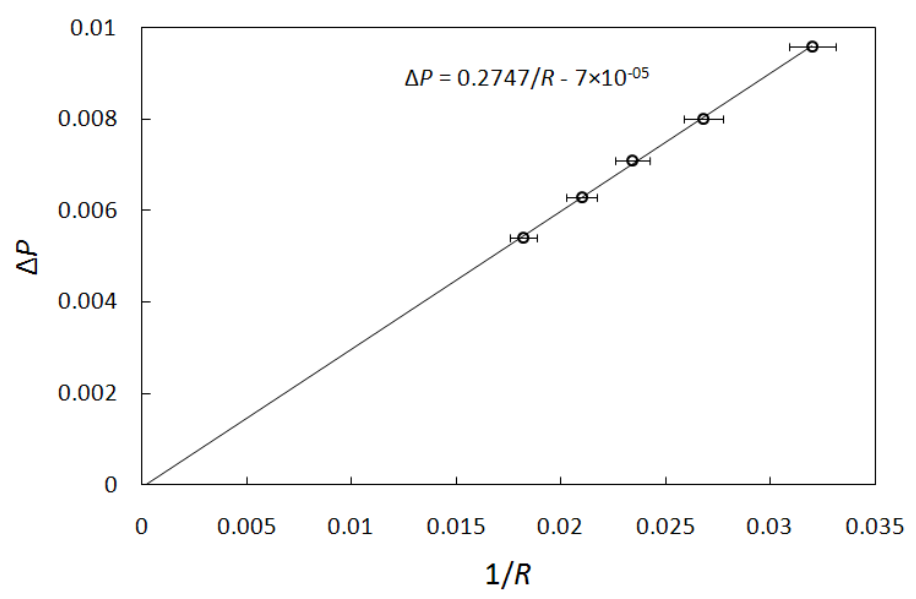

Figure 2: Verification of the Laplace law for droplets on the D2Q25 lattice.

To validate the realization of the Shan-Chen ELBM model on the D2Q25, a Laplace law test

$$
\Delta P=\frac{\sigma}{R}
$$

was conducted, by simulating different sized droplets and measuring the change in pressure, $\Delta P$, between the outside and inside of the droplet at equilibrium. The results are given in Fig. 2. The linear relationship between the pressure change and the inverse droplet radius $1 / R$ is a good verification of the accuracy of the model. One of the known issues with the Shan-Chen model is that the linear relationship is usually offset from the 
origin. For example, in a D2Q9 system at the same density ratio (and slightly different surface tension, due to the reference temperature difference), the equation of the line was found to be $y=0.2502 x+0.0003$. For the D2Q25 lattice this was $y=0.2747 x-0.00007$, with the intercept being significantly closer to zero.

A few comments on comparing the D2Q9 and the D2Q25 LB models are in order. To draw accurate comparisons between this system and the equivalent D2Q9 case, the parameters of the two systems should be matched as closely as possible. Surface tension, viscosity, density ratio and grid resolution all influence stability. The temperatures of the two systems differ, producing different surface tensions

$$
\sigma=\int_{-\infty}^{\infty}\left(P_{y y}-P_{x x}\right) d y=-\frac{G T_{0}^{2}}{2} \int_{-\infty}^{\infty}\left|\partial_{y} \psi\right|^{2} d y
$$

and viscosities

$$
\nu=T_{0}\left(\tau-\frac{1}{2}\right)
$$

The temperature in the D2Q9 system is $T_{0}=1 / 3$, while that in the low temperature D2Q25 model is $T_{0}=0.3675$. As this is only a small difference, the two systems can be compared fairly using the dimensionless Weber number. In systems where the temperature difference is larger it would be appropriate to alter the surface tensions to be equal.

One criticism of the Shan-Chen model is that surface tension and density ratio cannot be varied independently, both depending on the value of $G$. However, as introduced in Section 2.2, the grid refinement method allows density ratio and surface tension to be varied independently. The result of this is that grid resolution can be increased while keeping surface tension and density ratio the same, spreading the interface out over a larger number of grid points to increase stability. This method could be used to match the surface tension between the systems. However, in the case of the high reference temperature D2Q25 lattice used in the sequel, where $T_{0}=1.6325$, the grid size would have to be changed significantly to match parameters using grid refinement. This will therefore not be used in the results below, when comparing stability between the different systems. Changing the value of the relaxation time even by a small amount can result in large changes in stability at high density ratios, therefore the difference in viscosities will be accounted for by comparing Reynolds numbers. While this may make direct comparison between different systems difficult, trends in improvement of stability will still be clearly observable. Note that, in the framework of the Shan-Chen model, the viscosity of both the liquid and gas phases is the same and is given by Eq. (4.3), that is, apart from the relaxation time, it depends only on the reference temperature $T_{0}$. The corresponding speed of sound, $c_{\mathrm{s}}=\sqrt{T_{0}}$, is estimated as $c_{\mathrm{s}}=1.278$ for the D2Q25 lattice with the reference temperature $T_{0}=1+\sqrt{2 / 5}$. In the simulations below, the relative velocity of the droplets is considered up to $u=0.6$, which corresponds to a Mach number of $\mathrm{Ma}=u / c_{\mathrm{s}}<0.5$. Therefore, the simulated multi-phase flow can be still regarded as quasi-incompressible. 


\section{Combining methods and results}

We now have four methods available for improving the SC model, three of which (the grid refinement, the increased force term isotropy and the CS EoS) are known to individually make significant improvement to density ratios obtainable, while the higher-order D2Q25 lattice allows higher values of the velocity in a simulation. Both grid refinement and increased force term isotropy have been shown to independently reduce spurious velocities around stationary droplets, and the CS EoS has been shown to be capable of producing density ratios of over 1000, again for the stationary droplet case. We now look at the possibilities of combining some of these different methods, and using these combinations to look at binary droplet collisions.

We first look at combining a different EoS with other methods. It is important to note that when calculating the force term in each of the other three methods not only nearest neighbour points are used. For grid refinement next nearest neighbours are included in the calculation, for increased isotropy the number of points used increases with the desired order of isotropy, and the points used for the higher speed lattices depend on the lattice. The derivation for different EoS assumes nearest neighbours terms only are included. This assumption is not true for the three other methods, and it is therefore possible that combining EoS with these methods will not produce an improved result. Our tests have indeed shown that when the CS EoS is used with any of the other three methods, the density ratio, and Reynolds and Weber numbers achievable, are lower than when the EoS is used as the sole improvement to the SC model. Therefore, in the remainder of this work, the original SC EoS will be used. The three remaining methods have been tested in various combinations for the improvement of the stability of the Shan-Chen multiphase model-increased force term isotropy [3, 15], grid refinement [3], and higher velocity lattices [4]. The results from the two combinations of methods are given in Tables 1 and 2, along with those for the grid refinement alone, for comparison.

Table 1: Highest stable density ratio for droplet collision at $u=0.1$, for different combinations of methods of improving the basic Shan-Chen multiphase model.

\begin{tabular}{||l|c||}
\hline Method & Highest Density Ratio \\
\hline D2Q9: Grid refinement & 1840 \\
\hline D2Q9: Grid refinement with 14th order force term isotropy & 3590 \\
\hline D2Q25: Grid refinement & 2070 \\
\hline
\end{tabular}

Table 2: Highest stable Reynolds and Weber numbers for droplet collisions, at a density ratio of 1000, for different combinations of methods of improving the basic Shan-Chen multiphase model.

\begin{tabular}{||l|c|c||}
\hline Method & Highest Re & Highest We \\
\hline D2Q9: Grid refinement & 72 & 14 \\
\hline D2Q9: Grid refinement with 14th order force term isotropy & 123 & 31 \\
\hline D2Q25: Grid refinement & 71 & 38 \\
\hline
\end{tabular}




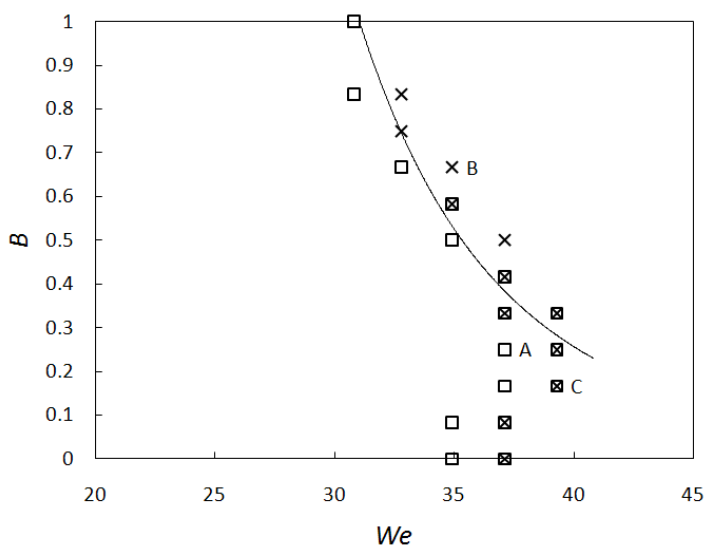

Figure 3: Coalescence (square), separation (cross) and intermediate regimes (crossed square) (separation followed by re-coalescence) for droplet collisions, using grid refinement and the D2Q25 lattice.

Highest density ratios were found for collisions with each droplet moving at $u=0.1$, by increasing the value of $G$, it should however be noted that differences in $T_{0}$ do give differences in surface tension and viscosity, and therefore comparison between density ratios are at slightly different Reynolds and Weber numbers. The initial lattice size used was $100 \times 100$. For the case of grid refinement, this was increased to $200 \times 200$, while keeping the properties of the system constant by setting $G_{2}=G / 2$ and $G_{1}=0$. This doubling of lattice size results in the halving of $\rho_{0}$.

Density ratio was then set to 1000 for all the models, and droplet velocity increased to find the highest stable Weber and Reynolds numbers. This provides a more accurate comparison between the methods, as dimensionless parameters are compared at equal density ratios.

It can be seen that the D2Q25 lattice has given an increase in attainable Weber number, when combined with grid refinement. Looking specifically at the comparison between the grid refinement method alone, and the grid refinement method with the D2Q25 lattice at the higher reference temperature, the highest stable Weber number is seen to increase by nearly a factor of three, at nearly the same Reynolds number. The higher Weber number is due to the much higher velocities, up to $u=0.6$, which can be stably simulated on the D2Q25 lattice (the Weber number increasing as the square of the relative velocity of the droplets), however there is a trade off from the increase in surface tension, due to the higher value of $T_{0}$. The Reynolds number is about the same at this higher velocity, due to the higher temperature increasing the viscosity of the system.

Using the grid refinement with the D2Q25 lattice, droplet collisions at high Weber numbers for varying impact parameters can be looked at in an attempt to reproduce the results of Fig. 1. The head on collision cases are more stable than for impact parameters approaching 1 . This is due to the separation that can occur, being less stable than the coalescence case. It was therefore necessary to decrease the density ratio slightly, to 500:1, to produce the results in Fig. 3. 


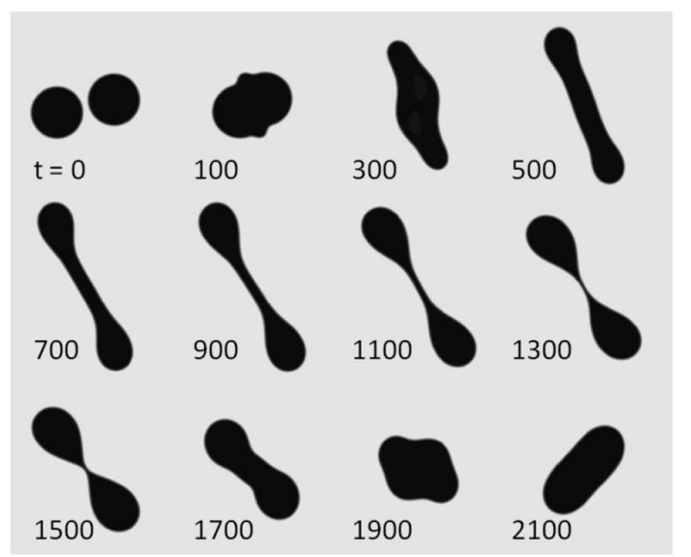

Figure 4: Lattice Boltzmann simulation of droplet coalescence, point " $\mathrm{A}$ " of Fig. 3, We $=37.0, B=0.25$, corresponding to Regime (III) of the experimental results in [9].

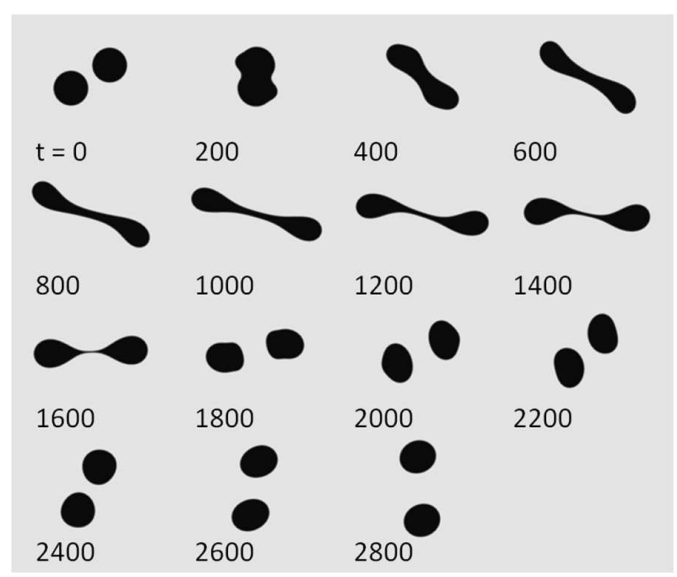

Figure 5: Lattice Boltzmann simulation of off-centre separation, point "B" of Fig. 3, We $=34.9, B=0.67$, corresponding to Regime (IV) of the experimental results in [9].

Points $A$ and $B$ in Fig. 3 corresponding to coalescence and separation, respectively, are shown in Fig. 4 and Fig. 5. Point $C$ shows an intermediate step observed between separation and coalescence, where the droplets separate briefly, but then re-coalesce, as shown in Fig. 6. The approximate line dividing coalescence and separation is qualitatively similar to that observed by Qian and Law [9]. It is possible that the transition from coalescence to head on separation is being observed at low impact parameters, however this requires a further investigation. In the current two-dimensional simulation, a fully reflexive separation was not observed, further investigation into this regime is required.

For a density ratio of 500:1, the highest stable Weber number reached was We $=127$. At this higher Weber number a different type of separation was observed, with droplets separating from either end, leaving a 3rd central droplet. This is shown in Fig. 7. This 


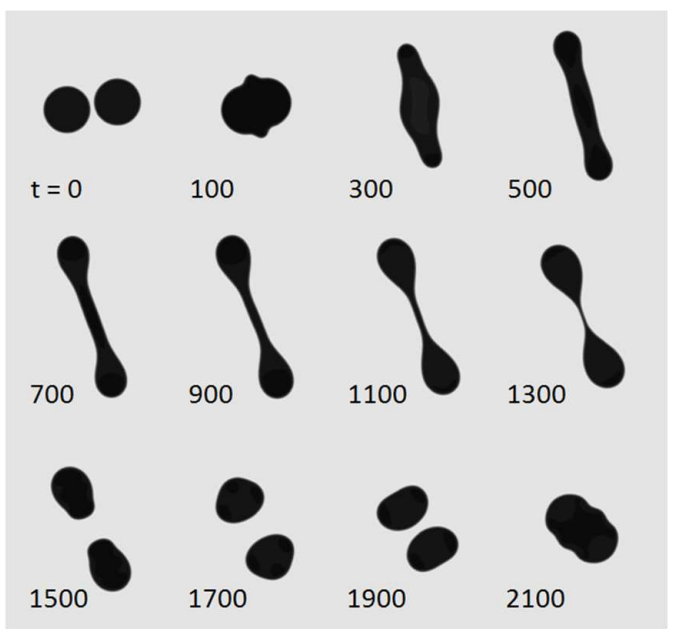

Figure 6: Droplet separation and re-coalescence, point " $C$ " of Fig. 3, We $=39.3, B=0.17$.

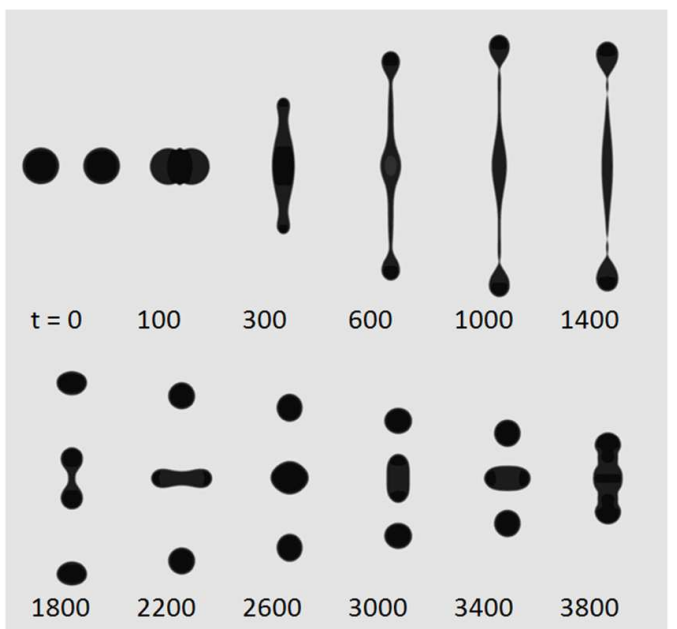

Figure 7: Head on separation and slow re-coalescence, $\mathrm{We}=127.2, B=0.0$.

head-on collision and separation case agrees qualitatively with experimental observations. In the present simulation the head on separation was followed by a slower process of re-coalescence which could be due to the two-dimensional feature of the LB model used. Clearly, more studies to clarify this point are needed in the future.

\section{Conclusions}

Starting from the Shan-Chen multiphase model, different improvements and their combinations have been considered. Spurious velocities around curved interfaces are known to cause instability, preventing the original Shan-Chen model from achieving high den- 
sity ratios. Methods that were previously known to reduce these velocities and therefore increase the density ratios achievable were re-examined. These included grid refinement [3], higher order force term isotropy $[3,15]$, and the inclusion of a more realistic EoS [2]. Finally, a new method for developing lattices with larger velocity sets was considered in detail and combined with the Shan-Chen model.

The density ratio of about 1000 required to simulate realistic multiphase systems has been achieved in different ways. The CS EoS was previously known to produce high density ratios, however it was found that due to the nearest neighbour assumption in the force term, this method could not be successfully combined with any of the other three. On the other hand, it has been shown that combining grid refinement with a higher velocity lattice or higher order isotropy in the force term can produce density ratios similar to those seen with the CS EoS. Future studies on using EoS when points beyond nearest neighbours need to be included in the force calculation are necessary.

At a fixed density ratio of 1000 , different combinations of methods were investigated. It has been shown that the multi-speed lattice allowed higher Weber numbers to be reached, by relaxing the constraint on velocities. When combined with grid refinement, the high-speed lattice can give roughly a factor of three increase in the Weber number over that possible with grid refinement alone. While Reynolds numbers remain low in all systems, this is a known problem with the single relaxation time LBM. Incorporating higher velocity lattices into a recently proposed quasi-equilibrium LBM [16] should allow higher Reynolds numbers to be reached, and will be considered in the future. It would also be worth studying further lattices derived from the entropic method, and looking at both their high and low temperature cases.

Finally the combination of the D2Q25 lattice with grid refinement was used for a preliminary study of the different droplets collision regimes for varying Weber number and impact parameter. This has shown good qualitative agreement with existing experimental results; however investigations at more realistic Reynolds numbers are required. Additionally the three-dimensional case needs to be considered, which will be possible using the recently introduced Galilean invariant lattice Boltzmann model [18].

\section{Acknowledgments}

We gratefully acknowledge the funding from the Engineering and Physical Sciences Research Council under the HEC Studentship scheme and grant No. EP/I000801/1.

\section{References}

[1] X. Shan, and H. Chen, Lattice Boltzmann model for simulating flows with multiple phases and components, Phys. Rev. E., 47 (1993), 1815-1819.

[2] P. Yuan, and L. Schaefer, Equations of state in a lattice Boltzmann model, Phys. Fluids., 18 (2006), 042101. 
[3] M. Sbragaglia, R. Benzi, L. Biferale, S. Succi, K. Sugiyama, and F. Toschi, Generalized lattice Boltzmann method with multirange pseudopotential, Phys. Rev. E., 75 (2007), 026702.

[4] S. S. Chikatamarla, and I. V. Karlin, Entropy and Galilean invariance of lattice Boltzmann theories, Phys. Rev. Lett., 9 (2006), 190601.

[5] S. S. Chikatamarla, and I. V. Karlin, Lattices for the lattice Boltzmann method, Phys. Rev. E., 79 (2009), 046701.

[6] X. He, and L.-S. Luo, Theory of the lattice Boltzmann method: from the Boltzmann equation to the lattice Boltzmann equation, Phys. Rev. E., 56 (1997), 6811-6817.

[7] N. Ashgriz, and J. Y. Poo, Coalescence and separation in binary collisions of liquid drops, J. Fluid. Mech., 221 (1990), 183-204.

[8] P. R. Brazier-Smith, S. G. Jennings, and J. Latham, The interaction of falling water drops: coalescence, Proc. R. Soc. London. A., 326 (1972), 393-408.

[9] J. Qian, and C. K. Law, Regimes of coalescence and separation in droplet collision, J. Fluid. Mech., 331 (1997), 59-80.

[10] T. Inamuro, T. Ogata, S. Tajima, and N. Konishi, A lattice Boltzmann method for incompressible two-phase flows with large density differences, J. Comput. Phys., 198 (2004), 628-644.

[11] A. J. Chorin, Numerical solution of the Navier-Stokes equations, Math. Comput., 22 (1968), $745-762$.

[12] K. H. Luo, J. Xia, and E. Monaco, Multiscale modelling of multiphase flow with complex interactions, J. Multiscale. Model., 1 (2009), 125-156.

[13] U. Frisch, D. d'Humires, B. Hasslacher, P. Lallemand, Y. Pomeau, and J.-P. Rivet, Lattice gas hydrodynamics in two and three dimensions, Complex. Syst., 1 (1987), 649-707.

[14] A. K. Gunstensen, D. H. Rothman, S. Zaleski, and G. Zanetti, Lattice Boltzmann model of immiscibel fluids, Phys. Rev. A., 43 (1991), 4320-4307.

[15] X. Shan, Analysis and reduction of the spurious current in a class of multiphase lattice Boltzmann models, Phys. Rev. E., 73 (2006), 047701.

[16] P. Asinari, and I. Karlin, Generalized Maxwell state and $H$-theorem for computing fluid flows using the lattice Boltzmann method, Phys. Rev. E., 79 (2009), 036703.

[17] N. F. Carnahan, and K. E. Starling, Equation of state for nonattracting rigid spheres, J. Chem. Phys., 51 (1969), 635-636.

[18] S. S. Chikatamarla, C. E. Frouzakis, I. V. Karlin, A. G. Tomboulides, and K.B. Boulouchos, Lattice Boltzmann method for direct numerical simulation of turbulent flows, J. Fluid. Mech., 656 (2010), 298-308. 